\title{
Carbon Monoxide in type II supernovae
}

\author{
J. Spyromilio, B. Leibundgut, and R. Gilmozzi \\ European Southern Observatory, Karl-Schwarzschild-Strasse 2, 85748 Garching, Germany \\ e-mail: bleibundgut@eso.org; rgilmozz@eso.org \\ Received 20 April 2001 / Accepted 27 June 2001

\begin{abstract}
Infrared spectra of two type II supernovae 6 months after explosion are presented. The spectra exhibit a strong similarity to the observations of SN 1987A and other type II SNe at comparable epochs. The continuum can be fitted with a cool black body and the hydrogen lines have emissivities that are approximately those of a Case B recombination spectrum. The data extend far enough into the thermal region to detect emission by the first overtone of carbon monoxide. The molecular emission is modeled and compared with that in the spectra of SN 1987A. It is found that the flux in the CO first overtone is comparable to that found in SN 1987A. We argue
\end{abstract} \\ that Carbon Monoxide forms in the ejecta of all type II SNe during the first year after explosion.
}

Key words. molecular processes - supernovae: individual: SN 1998dl - SN 1999em

\section{Introduction}

The presence of molecular emission in the spectra of supernovae while not new remains rarely detected. First detection of carbon monoxide in a supernova was in the spectra of SN 1987A (Catchpole \& Glass 1987; McGregor \& Hyland 1987; Ames Research Center 1987; Oliva et al. 1987; Spyromilio et al. 1988). In SN 1987A observations of the first overtone of $\mathrm{CO}$ at $2.3-\mu \mathrm{m}$ were complemented by observations at $4.6-\mu \mathrm{m}$ of the fundamental bands. In addition to carbon monoxide, the spectra of SN 1987A revealed bands of $\mathrm{SiO}$ (Meikle et al. 1993; Roche et al. 1991) and CS (Meikle et al. 1993). The presence of $\mathrm{H}_{3}^{+}$is also claimed in the spectra of SN 1987A (Miller et al. 1992) although alternative atomic species can also explain the identified features. Spyromilio \& Leibundgut (1996) reported the detection of $\mathrm{CO}$ first overtone emission in the spectra of the type II supernova 1995ad while Gerardy et al. (2000) and Fassia et al. (2001) reported the presence of CO in SN 1998S.

The formation of molecules in the ejecta of supernovae, even $\mathrm{CO}$ - the most stable of diatomic molecules with a dissociation energy of $11.09 \mathrm{eV}$ (Douglas \& Møller 1955), is not trivial. The high UV field due to recombinations and the energetic electrons from the radioactive decays of ${ }^{56} \mathrm{Ni}$ and its daughter ${ }^{56} \mathrm{Co}$ create an inhospitable environment with multiple dissociation paths (see Lepp et al. 1990).

Send offprint requests to: J. Spyromilio, e-mail: jspyromi@eso.org

* Based on observations collected at the European Southern observatory, Chile in time allocated to proposals $62 . \mathrm{H}-0708$ and 65.H.-0462.
The presence therefore of molecules in the ejecta imply a particular distribution of the material within the ejecta and place constraints on the degree of mixing possible. These effects have only been analyzed in detail in the well observed SN 1987A. The degree to which that object is representative of the entire class of type II SNe, which will almost by definition not be observed with the same accuracy, is of some importance.

Molecules also affect the degree of cooling of the ejecta. The partition function of a diatomic molecule such as $\mathrm{CO}$ is enormous compared to that of even a heavy ion such as $\mathrm{Fe}^{+}$. Moreover, the lower rotational levels of the fundamental, first and second overtones of $\mathrm{CO}$ require low energies for their excitations allowing them to continue cooling the ejecta even as the temperature drops.

Gerardy et al. (2000) and Fassia et al. (2001) also report the detection of emission from dust in SN 1998S and Fassia et al. argue that the cooling by CO may lead to conditions that favour the formation of dust.

Here we report on observations of two more type II SNe in the near infrared which exhibit emission by carbon monoxide. In Sect. 2 we report on the observations. The data are discussed and compared with those of SN 1987A and theoretical models in Sect. 3.

\section{Observations}

SN 1998dl in NGC 1084 (recession velocity $1411 \mathrm{~km} \mathrm{~s}^{-1}$ ) was discovered by King et al. (1998), on 1998 August 20, from observations made at the 0.8-m Katzman Automatic Imaging Telescope (KAIT). It was subsequently found to have been present in images taken on 1998, August 2 and 
August 9. Filippenko (1998) detected hydrogen Balmer lines in early optical spectra classifying the object as a type II supernova.

SN 1998dl was observed at the ESO 3.58-m New Technology Telescope on La Silla, on 1999 January 1, at an age of approximately 150 days. The near-infrared camera and spectrograph SofI (Moorwood et al. 1999) was used for these observations. The red low-resolution grism was used for the $H$ and $K$ band spectra. While the grism does cover the region between 1.8 and $1.92 \mu \mathrm{m}$ the atmospheric transparency is very low and no useful data are available in this region. A 1 arcsec slit was used which gives a resolution of $\lambda / \Delta \lambda$ of 650 and a wavelength coverage from $1.5 \mu \mathrm{m}$ to $2.5-\mu \mathrm{m}$. The spectroscopic standard BS 2290 (Allen \& Cragg 1983) was used to remove atmospheric features and to correct for the spectral response of the instrument. The conditions were not photometric and therefore the absolute fluxing of the spectrum is arbitrary. The slit projects to 3 pixels on the detector oversampling the data. Narrow features, of order 3 pixels (e.g. the particularly prominent feature at $2 \mu \mathrm{m}$ ), in the spectra are not real but result from incomplete corrections for atmospheric features.

Supernova 1999em in NGC 1637 (recession velocity $717 \mathrm{~km} \mathrm{~s}^{-1}$ ) was discovered by $\mathrm{Li}$ (1999) from observations made, on 1999 October 29, at the KAIT. Jha et al. (1999) and Deng et al. (1999) classified the object as a type II supernova based on spectroscopic observations.

SN 1999em was observed, on 2000 April 24, at an age of approximately 170 days with the same instrumental configuration as 1998dl. The spectroscopic standard used in this case was HIP 27855 (Perryman et al. 1997). The accurate removal of atmospheric features requires that the standard and the target are observed using the same resolving power. The usage of a narrow slit in both observations of the standard and the target implies that a separate photometric calibration of the data needs to be made to recover absolute fluxes. The imaging mode of SofI was used for this purpose and the NICMOS standard S121-E (Persson et al. 1998) was used for the calibration. The derived $K_{\mathrm{S}}$ magnitude of the supernova was 15.1 . The mean flux of the observed spectrum was corrected to this magnitude.

\section{Discussion}

The spectra of SN 1998dl and SN 1999em are displayed in Figs. 1 and 2, respectively. The data are shown at their observed wavelengths without correction for the recession velocity of the parent galaxies. The spectra are characterized by a strong continuum upon which emission lines are superimposed. The strong Brackett $\gamma$ line (rest wavelength $2.1655 \mu \mathrm{m}$ ) is evident. In the $\mathrm{SN} 1999 \mathrm{em}$ data the shorter wavelength transitions of the Brackett series can also be seen. Long-wards of the $\operatorname{Br} \gamma$ line, at $2.21 \mu \mathrm{m}$, a broad feature identified by Meikle et al. (1989) in the spectra of SN $1987 \mathrm{~A}$ as emission by the $2.207 \mu \mathrm{m} \mathrm{NaI} 4 \mathrm{~s}^{2} \mathrm{~S}-4 \mathrm{p}^{2} \mathrm{P}^{\circ}$ multiplet is evident ${ }^{1}$. Starting at $2.3 \mu \mathrm{m}$ and extending to the end of our spectral converage a broad feature is evident in the data. This broad feature we attribute to carbon monoxide first overtone transitions $(\Delta v=2)$. The much weaker second overtone $(\Delta v=3)$ is expected to start at $1.5 \mu \mathrm{m}$. Its contribution is expected to be only $1.4 \%$ of the strength of the first overtone (Bouanich \& Brodbeck 1974) and is therefore not detectable in our spectra. In much higher signal to noise data as would possibly be obtained at the VLT with ISAAC it might be possible to detect such emission. The fundamental $(\Delta v=1)$ band at $4.6 \mu \mathrm{m}$ is very much stronger than the first overtone observed here but also in an extremely unfavourable atmospheric window. This fundamental band, as mentioned in the introduction, almost dominates the cooling of the ejecta for some time and observations would be of great interest. Unfortunately they are probably limited to a combination of the very biggest telescopes and the very nearest supernovae.

A strong emission feature is observed at $2 \mu \mathrm{m}$. As noted above the narrow spike superimposed on the broad emission is instrumental. In SN 1987A, a broad feature at the same wavelength was identified by Meikle et al. as emission by the CaI $4 \mathrm{p}^{3} \mathrm{P}^{\circ}-3 \mathrm{~d}^{3} \mathrm{D}$ multiplet and the [FeI $] \mathrm{a}^{5} \mathrm{~F}_{5}-\mathrm{a}^{3} \mathrm{~F}_{4}$ multiplet. Assuming a contribution to the $2 \mu \mathrm{m}$ by $[\mathrm{FeI}]$ in the spectrum of SN 1998dl, the strong line at $1.51 \mu \mathrm{m}$ could be identified as emission by the $[\mathrm{FeI}] \mathrm{a}^{5} \mathrm{D}-2-\mathrm{a}^{5} \mathrm{~F}_{4}$ multiplet. Alternatively $\mathrm{MgI}$ also has a strong transition at $1.5031 \mu \mathrm{m}$. The lower recession velocity of the parent galaxy of SN $1999 \mathrm{em}$ prevents us from detecting the same line in that spectrum.

At $2.06 \mu \mathrm{m}$ both in SN 1998dl and SN 1999em a small absorption is present. This we identify as the P-Cygni trough of the HeI $2 \mathrm{~s}^{1} \mathrm{~S}-2 \mathrm{p}^{1} \mathrm{P}^{\circ} 2.058 \mu \mathrm{m}$ transition. We note that this region is one of poor atmospheric transmission with a strong telluric $\mathrm{CO}_{2}$ feature at $2.06 \mu \mathrm{m}$. While we believe that we have correctly compensated for this it is possible that the feature we identify as due to HeI is due to poor cancellation of the aforementioned atmospheric feature. HeI is expected to be very weak but its presence would be significant as highly excited He is a tracer for the presence of energetic electrons from the radioactive decay (Graham 1988; Fassia \& Meikle 1999). The singlet transition observed here would need to develop sufficient optical depth to exhibit a P-Cygni trough. While transitions from $2 \mathrm{~s}^{1} \mathrm{~S}$ to the ground state of HeI are forbidden, depopulation of this energy level can occur via two photon decay with a low transition probability $\left(A=51 \mathrm{~s}^{-1}\right)$. Given the age of the supernovae at the time of observation even this process would have de-populated the ground state of the $2.058 \mu \mathrm{m}$ line. Recombinations are the obvious source of electrons which, as the transitions from the ${ }^{1} \mathrm{P}^{\circ}$ states to the ground are expected to be saturated, will naturally cascade down to the $2 \mathrm{~s}^{1} \mathrm{~S}$ level. Given the high ionization potential of $\mathrm{He}(24.587 \mathrm{eV})$ the presence of the $2.058 \mu \mathrm{m}$

${ }^{1}$ In Meikle et al. (1989) the lower level of this multiplet in Table 3 is incorrectly labeled as $4 \mathrm{~s}^{2} \mathrm{D}$. 


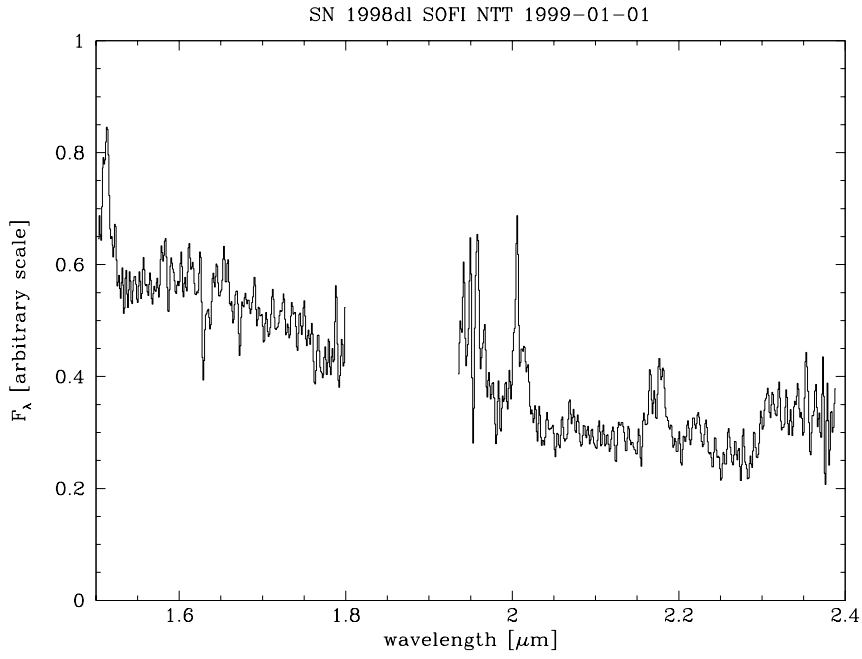

Fig. 1. Spectrum of 1998dl.

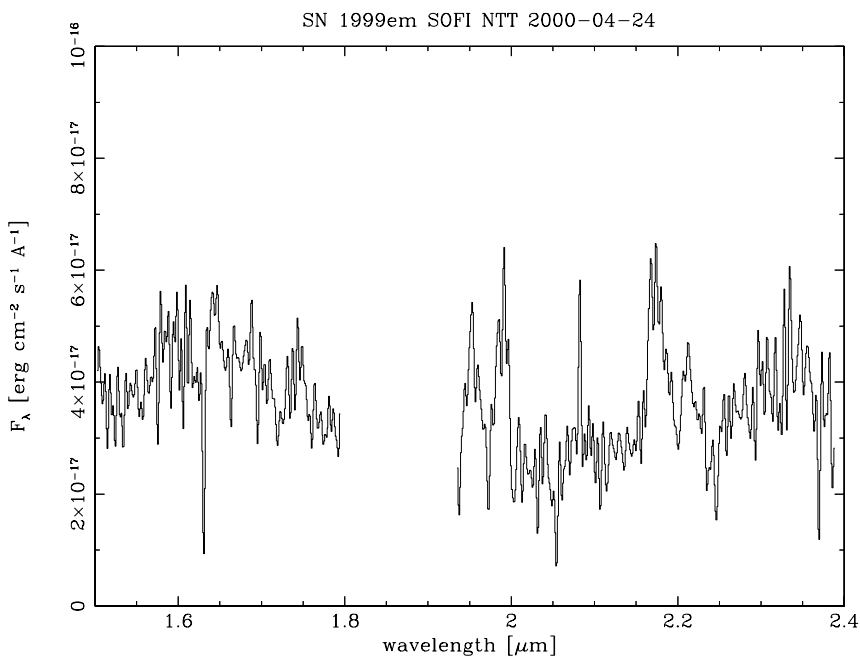

Fig. 2. Spectrum of 1999em.

transition suggests that the excitation by energetic electrons argued by Graham and Fassia \& Meikle is occuring also in these two SNe.

We have used simple line models to make fits to the data. These fits are shown in Figs. 3 and 4. The ingredients of the models are described below.

The continuum in the models is a black-body with a temperature of $3300 \mathrm{~K}$ for SN 1998dl and $2000 \mathrm{~K}$ for SN $1999 \mathrm{em}$. The flux observed in the spectrum of SN 1999em at this temperature corresponds to a surface of last scattering expanding at $500 \mathrm{~km} \mathrm{~s}^{-1}$ for 170 days at a distance of 7.8 Mpc (Sohn \& Davidge 1998).

For the carbon monoxide a simple LTE (Boltzmann distribution) model of the populations of the rotationalvibrational energy levels in the ground state electronic level ${ }^{1} \Sigma$ of ${ }^{12} \mathrm{C}^{16} \mathrm{O}(\mathrm{CO})$ was used. This model has been described before in Spyromilio et al. (1988). Briefly, the energy levels for a given rotational level $J$ and a given vibrational level $v$ can be determined using:

$$
\begin{aligned}
E= & \omega\left(v+\frac{1}{2}\right)-\omega x\left(v+\frac{1}{2}\right)^{2}+\omega y\left(v+\frac{1}{2}\right)^{3} \\
& +B_{v} J(J+1)-D_{v} J^{2}(J+1)^{2}
\end{aligned}
$$

with $\omega, \omega x, \omega y, B_{v}$ and $D_{v}$ from Young (1968). The $A$ values are given by

$A_{v v^{\prime}}=\frac{64 \pi^{4} \nu^{3}}{3 h g_{J}}(T M)^{2}$

where $\nu$ is the transition frequency, $g_{J}=2 J+1$ and $T M$ is the transition matrix from vibrational level $v$ to $v^{\prime}$. TM for $\Delta J$ of \pm 1 is given by Chackerian \& Tipping (1983). The lowest 15 vibrational levels were included and within each 110 rotational level populations were calculated.

As has been shown in the spectra of SN 1987A (Spyromilio et al. 1988) the CO band heads are sensitive to the excitation temperature. Higher temperature in the CO model increase the contribution of higher vibrational pairs and increase the emission long-wards of the $v=2$ to $v^{\prime}=0$ band-head. The quality of the data is not good enough to reliably distinguish between small changes in the temperature. The SN 1998dl data indicate a slightly higher temperature than the SN $1999 \mathrm{em}$. The fits use $2500 \mathrm{~K}$ for SN 1998dl and $2000 \mathrm{~K}$ for SN $1999 \mathrm{em}$. The uncertainty in these values is of order a few hundred degrees $\mathrm{K}$. The difference in the temperatures leads to a significantly different appearance of the first overtone emission. The $2000 \mathrm{~K}$ temperature for the SN $1999 \mathrm{em}$ spectrum is based on the assumption that the drop in flux long-wards of $2.35 \mu \mathrm{m}$ is real. The SN 1998dl spectra appear to show a much smoother decline towards longer wavelengths.

The velocity at which the CO is expanding can be used to constrain the mass of the progenitor (Gerardy et al. 2000). In SN 1998S Gerardy et al. derive a high expansion velocity $\left(v \approx 3000 \mathrm{~km} \mathrm{~s}^{-1}\right)$ based on the smooth rise of the 2-0 $R$-branch band head and a progenitor mass in excess of $25 M_{\odot}$. Fassia et al. (2001) do not detect such a steep rise and argue for a lower velocity. In addition their modeling of the ejecta favours a lower mass progenitor. We argue below in favour of the low range of velocities although we, like Gerardy et al., have a smooth rise of the 2-0 R-branch band head.

In the spectra of SN 1987A a rigorous fitting of the expansion velocity could be made (Spyromilio et al. 1988) based on the appearance of the $R$-branch emission from each pair of energy states. Both Gerardy et al. (2000) and Fassia et al. (2001) use the lack of clear separation of the $R$-branch band heads to place lower limits on the expansion velocity in the observations of SN 1998S. This same effect can be used by us to place a weak lower limit of $1000 \mathrm{~km} \mathrm{~s}^{-1}$ on the expansion velocity.

The comparison of SN 1998S with SN 1987A (see Gerardy et al. Fig. 6) shows that another line is possibly blended with the $\mathrm{CO}$ band head at $2.28 \mu \mathrm{m}$. This feature, which at the recession velocity of SN 1987A is observed at $2.26 \mu \mathrm{m}$, is discussed later but remains unidentified. The $2.26 \mu \mathrm{m}$ feature is very strong in the spectra of 
SN 1999em and weakly present in our data on SN 1998dl. The same feature was present in the spectra of SN 1995ad (Spyromilio \& Leibundgut 1996). We cannot account for the lack of detection of this in the data of Fassia et al. (2001). The blending of this feature into the band head implies that models that do not take this into account will underestimate the true sharpness of the band head rise and derive higher than expected expansion velocities. The best estimate of the velocity of the $\mathrm{CO}$, we believe, comes from the comparison of the observed spectra with those of SN 1987A (see Figs. 5 and 6). The good matching of the rise of the 2-0 band head suggests that the velocity observed in SN 1987A (1800-2000 $\mathrm{km} \mathrm{s}^{-1}$; Spyromilio et al. 1988) is representative of the velocity of the CO in SN 1998dl and SN 1999em. Clearly in the absence of the blended line we would agree with Gerardy et al. (2000) and also derive a much higher expansion velocity for the CO. Each CO line in our models was convolved with a Gaussian line profile of $F W H M 2000 \mathrm{~km} \mathrm{~s}^{-1}$.

The Brackett series line strengths in the model assume a case-B recombination spectrum at a temperature of $3000 \mathrm{~K}$ and electron density of $10^{5}$ (Hummer \& Storey 1987). The $\operatorname{Br} \gamma$ and $9 \rightarrow 4$ transitions are well fit using these parameters. The $\operatorname{Br} \delta$ line lies at the very edge of the atmospheric window and the cancellation of the atmospheric features there is poor. While the norm would be not to display the spectra in this region due to the large uncertainties in the data we include them here. The data show that the spectra are consistent with the model. We do not derive any further information from the region around $1.92 \mu \mathrm{m}$. Unfortunately the $\mathrm{Br} \epsilon$ transition lies in the gap between the two atmospheric windows and is not observable from the ground. The FWHM of the Gaussian line profile computed was $3000 \mathrm{~km} \mathrm{~s}^{-1}$. As has been the case in the other SNe in which CO was detected, the velocity of the hydrogen lines is higher than that of the CO.

The individual lines identified earlier as NaI, CaI and [FeI] have not been included in the displayed fits as the individual contributions of the species would be arbitrary.

As already discussed with respect to the expansion velocity, the observations presented here can be compared with the observations of SN 1987A (data from Meikle et al. 1989). Figure 5 shows the data from SN 1987A taken at an age of 192 days superimposed on the spectra of SN 1998dl. The SN 1987A data have been scaled to match the flux in the $\mathrm{CO}$ feature and red-shifted to the recession velocity of NGC 1084. The continuum underlying the SN 1987A data has been artificially adjusted to match the average continuum level in the SN 1998dl data. The SN 1998dl continuum is much bluer than that of SN 1987A at the epoch at which the comparison data are taken. The poor correlation at $2.25 \mu \mathrm{m}$ is partially due to this effect and partially due to the difference in the strength of the $2.26 \mu \mathrm{m}$ feature. Given the quality of the SN 1998dl data, this comparison, adds plausibility to the identification of $\mathrm{CO}$ in the spectra. Also as mentioned above it allows a more accurate determination of the velocity structure of $\mathrm{CO}$ than can be achieved from the models. A similar comparison is made

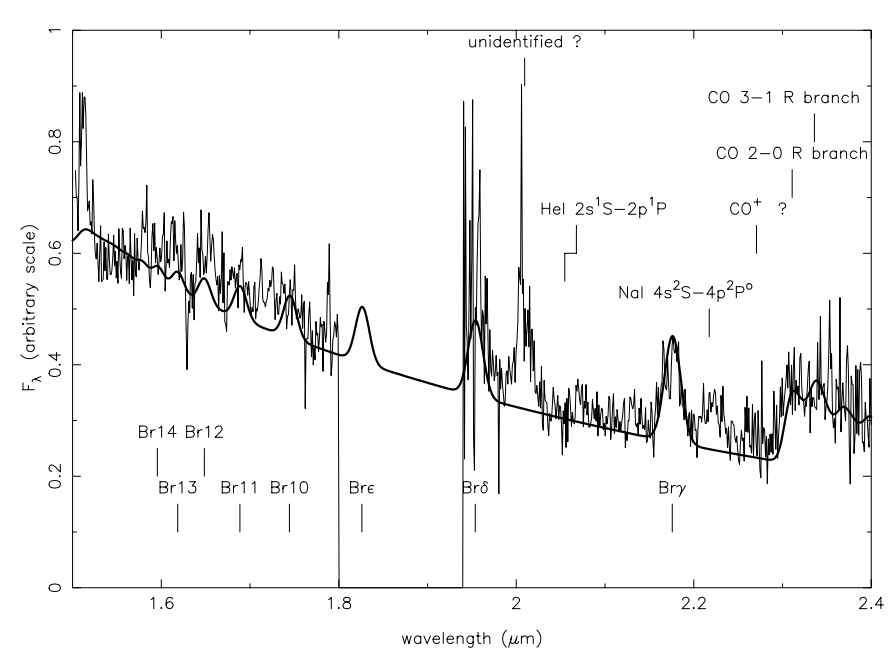

Fig. 3. Spectrum of $1998 \mathrm{dl}$ and model. Features discussed in the text are identified in the figure.

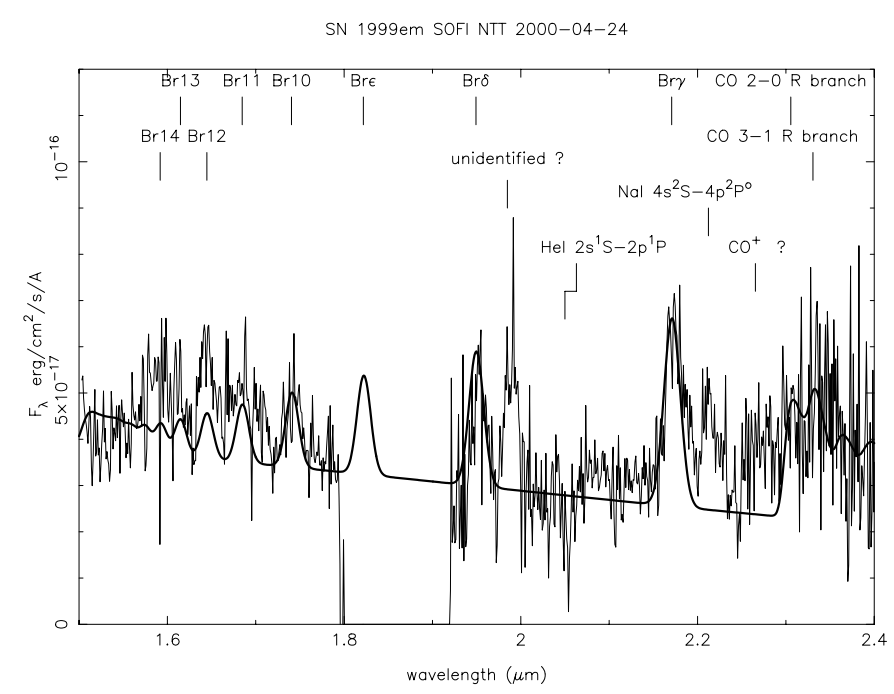

Fig. 4. Spectrum of 1999em and model. Features discussed in the text are identified in the figure.

in Fig. 6 for SN 1999em using SN 1987A data obtained at an age of 255 days.

The flux within the CO emission band for SN 1999em is $5.5 \times 10^{-11} \mathrm{ergs} \mathrm{s}^{-1} / \mathrm{cm}^{2}$ which at the distance of NGC 1637 (7.8 Mpc; Sohn \& Davidge 1998) is 20\% lower than that of SN 1987A at a comparable epoch.

The mass that corresponds to this emission depends critically on the emission model. Our LTE model gives masses of order $10^{-4} M_{\odot}$. Liu \& Dalgarno (1995) predict a much higher mass $\left(\sim 10^{-3} M_{\odot}\right)$ for the same emission at this age and argue that the conditions within the ejecta favour the formation of this amount of $\mathrm{CO}$. In fact it is expected that significantly more $\mathrm{CO}$ formed during the youth of the supernova $(\approx 100$ days after explosion) but has been destroyed. Gearhart et al. (1999) favour the formation of much less CO than Liu \& Dalgarno and more in line with our mass estimates. Both Gerardy et al. (2000) and Fassia et al. (2001) employ non-LTE and 
SN 1998dl SOFI NTT 1999-01-01 \& SN 1987A at 192 days

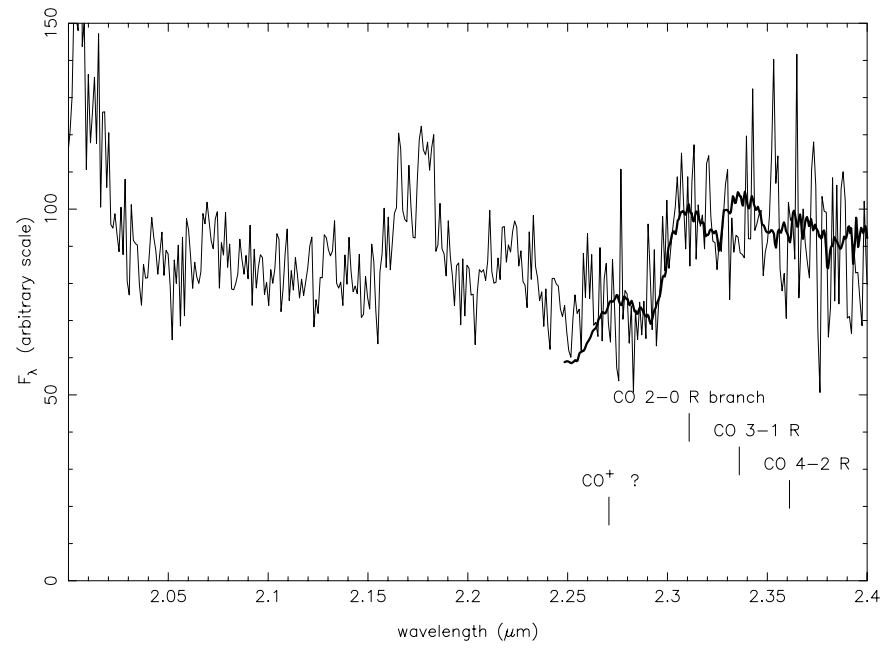

Fig. 5. Spectrum of 1998 dl in the $K$ band with spectrum of SN1987A superimposed.

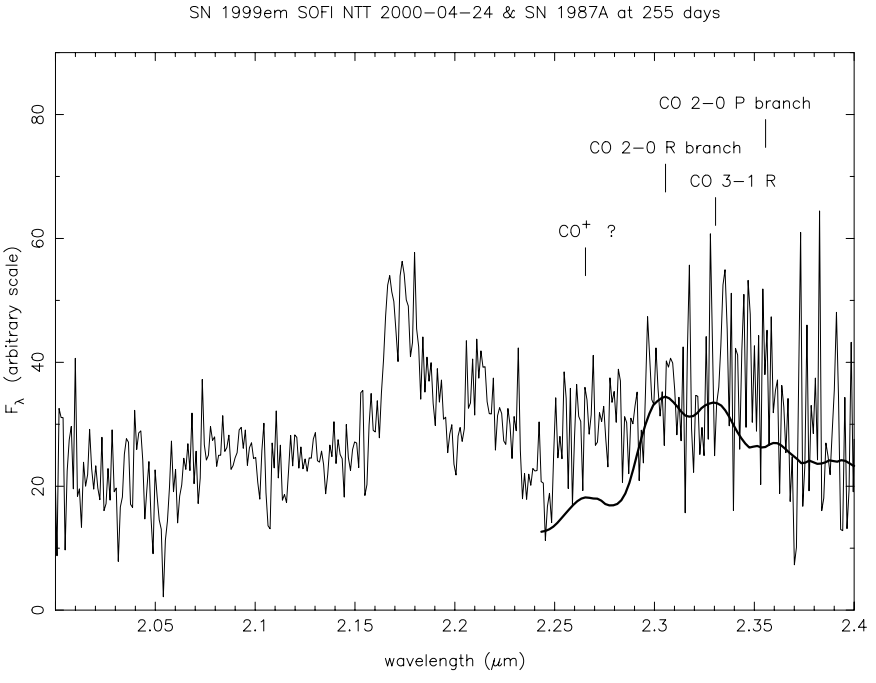

Fig. 6. Spectrum of $1999 \mathrm{em}$ in the $K$ band with spectrum of SN1987A superimposed. Note that the feature marked as $\mathrm{CO}^{+}$ is much weaker in $87 \mathrm{~A}$ than in $99 \mathrm{em}$.

optically thick models to estimate the mass of $\mathrm{CO}$ emitting and derive high masses. These models base their input on assumptions and parameters that cannot be derived from our limited observations and even in the case of SN 1998S are based on assumed filling factors. Moreover Gerardy et al. argue that their comparisons are more a proof of principle rather than true models. We restrict ourselves therefore to our comparison with SN 1987A. Complications due to clumping and asphericity are beyond the scope of this work to address but it is worth noting that polarization has been detected in the spectra of SN 1999em (Leonard et al. 1999; Wang et al. 1999).

Sollerman et al. (2001) detected no sign of dust in spectroscopic observations of SN $1999 \mathrm{em}$ obtained at an age of 450 days. While the formation of molecules may provide the cold sites for dust formation in the ejecta of type II supernovae and therefore may be a necessary precondition, it is clearly not sufficient.

The feature at $2.26-\mu \mathrm{m}$ short-wards of the $\mathrm{CO}$ $v=2 \rightarrow 0$ band head remains unidentified. The formation of sufficient $\mathrm{CO}^{+}$, whose first overtone $v=2 \rightarrow 0$ band head coincides with this feature, is excluded by both Gearhart et al. and Liu \& Dalgarno. Note, however, that Petuchowski et al. (1989) have argued that $\mathrm{CO}^{+}$could form in sufficient quantities. The feature was reported by and identified as $\mathrm{CO}^{+}$by Spyromilio et al. (1988) and reported by Spyromilio \& Leibundgut in the spectra of SN 1995ad. It is also observed by Gerardy et al. (2000) in SN 1998S. Fassia et al. (2001) did not detect the emission in their spectra of SN 1998S. While theoretical models fail to predict the necessary amounts of $\mathrm{CO}^{+}$the identification of this feature remains insecure.

\section{Conclusions}

The spectra presented in this paper combined with the observations presented by Spyromilio \& Leibundgut (1996), Gerardy et al. (2000) and Fassia et al. (2001) suggest that the formation of $\mathrm{CO}$ in the ejecta of type II supernovae is ubiquitous at an age of 3 to 6 months.

The mass of $\mathrm{CO}$ present in the ejecta is difficult to estimate accurately. Our LTE models while simplistic rely on few assumptions with respect to the distribution and excitation of the CO. While for well studied supernovae such as SN 1987A the much more detailed models of Lepp et al. (1990) are well justified, it is arguable whether the input parameters for such models are well enough constrained in the case of the objects described here. We therefore argue that since the absolute fluxes argee for SN 1987A and SN $1999 \mathrm{em}$ so, to first approximation, will the emitting masses.

The expansion velocity of the $\mathrm{CO}$ is not accurately determined. By comparison with SN 1987A we argue that velocities around $2000 \mathrm{~km} \mathrm{~s}^{-1}$ are consistent with our data. We also argue that the blending of the ubiquitous (in our data) feature at $2.26 \mu \mathrm{m}$ with the $2-0 R$ branch band head has to be taken into account when using this profile for velocity determinations.

We note that comparing the spectra of the four other supernovae with CO emission with those of SN 1987A it is consistently the case that the spectral signature of the emission resembles that of SN 1987A at a later age than that at which the other SNe are observed.

The other aspects of our data indicate that the spectra of type II SNe in the near IR are very similar and that recombination continues to play a major role even 5 to 6 months after explosion.

A better understanding of the physical conditions in the ejecta of supernovae can be achieved with higher quality infrared data as would be obtained with ISAAC at the VLT. Very high quality data may even constrain the isotopic ratios of carbon and oxygen as the wavelengths of the band heads to shift depending on the exact isotopes. 
Acknowledgements. We thank the staff at the NTT for their excellent support over many years of observing. We thank D. Silva for outNEDing NED. This research has made use of the NASA/IPAC Extragalactic Database (NED) which is operated by the Jet Propulsion Laboratory, California Institute of Technology, under contract with the National Aeronautics and Space Administration.

\section{References}

Allen, D. A., \& Cragg, T. A. 1983, MNRAS, 203, 777

Ames Research Center, \& Cohen, M. 1987, IAU Circ., 4500

Bouanich, J. P., \& Brodbeck, D. J. 1974, J. Quant. Spectrosc. Rad. Tranf., 14, 1199

Catchpole, R., \& Glass, I. 1987, IAU Circ., 4457

Chakerian, C. Jr., \& Tipping, R. H. 1983, J. Mol. Spectrosc., 99, 431

Deng, J. S., et al. 1999, IAU Circ., 7296

Douglas, A. E., \& Møller, C. K. 1955, Can. J. Phys., 33, 125

Fassia, A., \& Meikle, W. P. S. 1999, MNRAS, 302, 314

Fassia, A., et al. 2001, MNRAS, 325, 907

Filippenko, A. V. 1998, IAU Circ., 6994

Gearhart, R. A., Wheeler, J. G., \& Swartz, D. A. 1999, ApJ, 510,944

Gerardy, C. L., Fesen, R. A., Hoeflich, P., \& Wheeler, J. C. 2000, AJ, 119, 2968

Graham, J. R. 1988, ApJ, 335, L53

Hummer, D. G., \& Storey, P. J. 1987, MNRAS, 224, 801

Jha, S., Challis, P., Garnavich, P., \& Kirshner, R. P. 1999, IAU Circ., 7296
King, J. Y., et al. 1998, IAU Circ., 6992

Sollerman, et al. 2001, in preparation

Leonard, D. C., Filippenko, A. V., \& Chornock, R. T. 1999, IAU Circ., 7305

Lepp, S., Dalgarno, A., \& McCray, R. 1990, ApJ, 358, 262

Li, W. D. 1999, IAU Circ., 7294

Liu, W., \& Dalgarno, A. 1995, 454, 472

McGregor, P. J., \& Hyland, A. R. 1987, IAU Circ., 4468

Meikle, W. P. S., Allen, D. A., Spyromilio, J., \& Varani, G. -F. 1989, MNRAS, 238, 193

Meikle, W. P. S., Spyromilio, J., Allen, D. A., Varani, G. -F., \& Cumming, R. J. 1993, MNRAS, 261, 535

Miller, S., Tennyson, J., Lepp, S., \& Dalgarno, A. 1992, Nature, 355,420

Moorwood, A., Cuby, J.-G., \& Lidman, C. 1998, ESO Messenger, 91, 9

Oliva, E., Moorwood, A. F., \& Danziger, I. J. 1987, IAU Circ., 4484

Perryman, M. A. C., et al. 1997, A\&A, 323, L49

Persson, S. E., Murphy, D. C., Krzeminski, W., Roth, M., \& Rieke, M. J. 1998, AJ, 116, 2475

Petuchowski, S. J., Dwek, E., Allen, J. E. Jr., \& Nuth, J. A. III 1989, ApJ, 342, 406

Roche, P. F., Aitken, D. K., \& Smith, C. H. 1991, MNRAS, $252,29 \mathrm{P}$

Sohn, Y.-J., \& Davidge, T. J. 1998, AJ, 115, 130

Spyromilio, J., Meikle, W. P. S., Allen, D. A., \& Learner, R. C. M. 1988, Nature, 334, 327

Spyromilio, J., \& Leibundgut, B. 1996, MNRAS, 283, L89

Wang, L., Baade, D., Ma, F., et al. 2000, IAU Circ., 7355

Young, L. A. 1968, J. Quant. Spectrosc. Rad. Tranf., 8, 693 